\title{
Technical Points Regarding New Enterostomy Formation for Incarcerated Stomal Prolapse in Loop Enterostomy
}

\author{
Tadao Okada ${ }^{1}$, Shohei Honda ${ }^{1}$, Hisayuki Miyagi ${ }^{1}$, Akinobu Taketomi ${ }^{2}$ \\ ${ }^{1}$ Department of Pediatric Surgery, Hokkaido University Hospital, Sapporo, Japan \\ ${ }^{2}$ Department of Gastroenterological Surgery I, Hokkaido University \\ Graduate School of Medicine, Sapporo, Japan \\ E-mail: okadata@med.hokudai.ac.jp \\ Received May 11, 2011; revised July 15, 2011; accepted September 6, 2011
}

\begin{abstract}
Aim: Incarcerated stomal prolapse is a rare complication of enterostomy. Numerous procedures have been described, such as additional laparotomy to fix the intraabdominal intestine in place, enterostomy revision, or correction of the prolapse following stoma creation. The authors report successful managements by stomal reconstruction and discuss several clinical points, including the techniques of surgical revision for incarcerated stomal prolapse in loop enterostomy. Patients: Case 1) A female infant weighing $2755 \mathrm{~g}$ was delivered at 34 weeks of gestation. On the first day after birth, a right supra-abdominal transverse incision of $10 \mathrm{~cm}$ in diameter was used for transverse loop colostomy in a cloacal malformation. Two centimeters of the stomal loop was approximated with sutures to prevent evisceration of the small intestine between the 2 limbs of the loop. Interrupted sutures of 5-0 absorbable monofilament secured the seromuscle of the colon to the peritoneum and fascia, and also to the skin. The distal limb of the colostomy prolapsed 11 months after birth. The physical findings revealed that $10 \mathrm{~cm}$ of the distal limb was intussuscepted. Case 2) A female infant weighing $2550 \mathrm{~g}$ was delivered at 39 weeks of gestation. A radiological examination by contrast enema showed no spastic rectum and colon, as in Hirschsprung's disease. Under the laparotomy of a right supra-abdominal transverse incision of $5 \mathrm{~cm}$ in diameter, loop ileostomy was performed at $30 \mathrm{~cm}$ on the proximal side of the cecum such as Case 1. Subsequently, the proximal limb of the ileostomy prolapsed 2 days after operation. The physical findings revealed that $10 \mathrm{~cm}$ of the proximal limb was intussuscepted. New enterostomy formation: Divided enterostomy was performed with $3-\mathrm{cm}$ stitching of each limb. The stomal site was moved to the inside from the previous stomal site to oversew and fix by the rectal fascia. The children have been well without trouble since undergoing the new eneterostomy formation. Conclusions: Operation to repair the prolapse of a stoma is advised if it causes problems. We found that simple mobilization of the bowel and excision of the redundant bowel provided a satisfactory result in the present cases.
\end{abstract}

Keywords: Incarcerated Stomal Prolapse, Stomal Reconstruction, Loop Enterostomy, Divided Enterostomy

\section{Introduction}

Intestinal stomal formation in infants and children is a common procedure undertaken as part of the surgical management of congenital malformations and acquired conditions of the gastrointestinal tract [1]. Complications of stoma have been decreasing recently due to improvements in both surgical techniques and the stoma care system. However, we sometimes still encounter patients who have stoma problems. The placement of a stoma for the diversion of intestinal flow in children, particularly in neonates, may present problems because a very dilated bowel must be brought through a thin abdominal wall [2]. Stomal prolapse occurs rather frequently with loop stomas, but incarceration is rare [2,3]. Both proximal and distal parts of the limbs of the stoma can prolapse, which can lead to ischemia of the stoma or of the extruded bowel [4]. Numerous procedures have been described, such as additional laparotomy to fix the intraabdominal colon in place [2,5], enterostomy revision, or correction of the prolapse following stoma creation [4]. Golladay, et al. [2] reported that creation of an end loop stoma by 
the purse string technique prevented prolapse and could be accomplished expeditiously. However, occasionally, the stoma must be revised [6]. Management strategies we have employed include operating on one patient with incarcerated stomal prolapse.

We describe herein 2 infants: first, an infant with a cloacal anomaly who underwent reconstruction for incarcerated stomal prolapse in a loop colostomy; second, an infant with Hirschsprung's disease who underwent reconstruction for incarcerated stomal prolapse in a loop ileostomy. After reviewing other cases, we discuss several special operative techniques regarding new stomal formation for incarcerated stomal prolapse in loop colostomy and ileostomy, especially with regard to the suspected causes of stomal prolapse.

\section{Case Report}

\subsection{Case 1}

A female infant weighing $2755 \mathrm{~g}$ was delivered by normal vaginal delivery at 34 weeks of gestation. Prenatal ultrasonography (US) had not disclosed any abnormal findings. Immediately after birth, severe abdominal distention became evident. On physical examination, a single perineal opening implied the presence of a cloacal malformation. Laboratory examinations revealed neither anemia nor signs of inflammation. A plain radiograph disclosed multiple bubbles in the intestine. Abdominal US disclosed right hydrosalpinx. On the first day after birth, a right supra-abdominal transverse incision of 10 $\mathrm{cm}$ in diameter was used for the drainage of the right fallopian tube and transverse loop colostomy. Two centimeters of the stomal loop was approximated with sutures to prevent evisceration of the small intestine between the 2 limbs of the loop. Triangular stitches were made to the rectal fascia and peritoneum, and to each limb of the colonic loop at the point where the 2 limbs of the loop were in contact with one another. Interrupted sutures of 5-0 absorbable monofilament secured the seromuscle of the colon to the peritoneum and fascia, and also to the skin. To avoid tension on the underlying suture line, a small rubber tube was placed under the loop for 5 days.

A cystocutaneostomy was performed 2 months after birth because of right vescicoureteral reflux with relapsing urinary tract infections. Thereafter, right ureterocutaneostomy was performed 8 months after birth. She had neither sacral neural anomalies nor disturbances which affected abdominal muscle tone.

The distal limb of the colostomy prolapsed 11 months after birth. The physical findings revealed that $10 \mathrm{~cm}$ of the distal limb was intussuscepted, and the color of the intussuscepted colon was dark-brown (Figure 1). The patient could not undergo early colostomy closure because her body size was too small for a radical operation to repair the cloacal anomaly. Manual, gentle reduction of the intussuscepted colon was successful, and the patient was prepared for reconstruction of the stoma by the administration of oral antibiotics and fasting for $24 \mathrm{hr}$ before the surgery. The stomal opening was two-fingers wide.

For reconstruction of the stoma, a transverse abdominal incision near the medial angle of the stoma was placed in the previous wound 7 days after the manual reduction. There was little abdominal ascites and the suture fixation to each limb of the colonic loop was loose (Figure 2). Ileocecal resection was required for stoma-revision of the divided colostomy because the colostomy had been placed in the 4-cm oral portion of the ascending colon from the appendix on the basis of operative findings. Divided colostomy was performed with $3-\mathrm{cm}$ stitching of each limb (Figure 2). The stomal site was moved to the inside from the previous stomal site to oversew and fix by the rectal fascia (Figure 2). Fluids were administered by mouth for $48 \mathrm{hr}$, and thereafter, milk feedings were resumed. The child has been well without trouble for 3 years from stomal reconstruction.

\subsection{Case 2}

A female infant weighing $2550 \mathrm{~g}$ was delivered by normal vaginal delivery at 39 weeks of gestation. She evacuated once a week at 6 months after birth, and vomiting appeared at 11 months old. She was referred to our institute for further evaluation of constipation and vomiting at 1 year old. Neither sacral neural anomalies nor disturbances which affected abdominal muscle tone were noted.

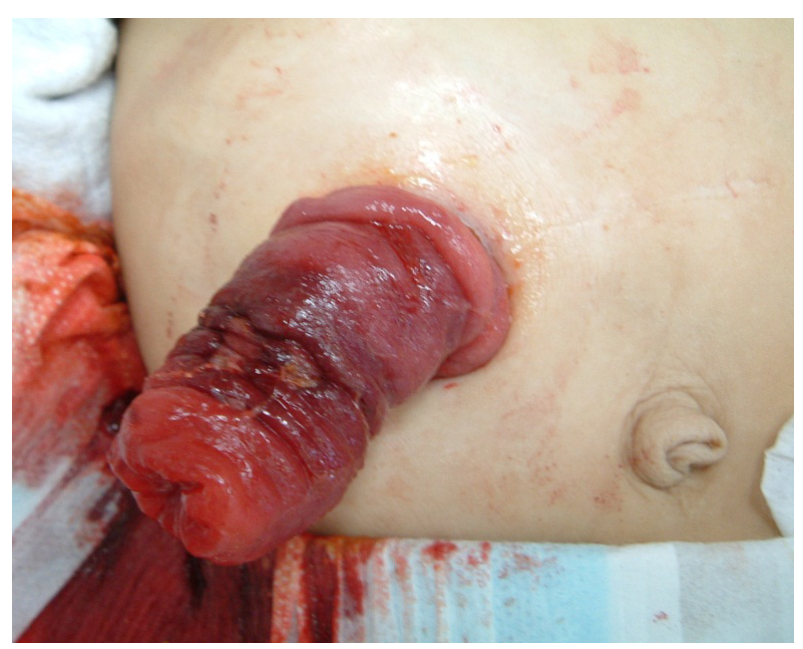

Figure 1. Appearance of incarcerated stomal prolapse in Case 1. 


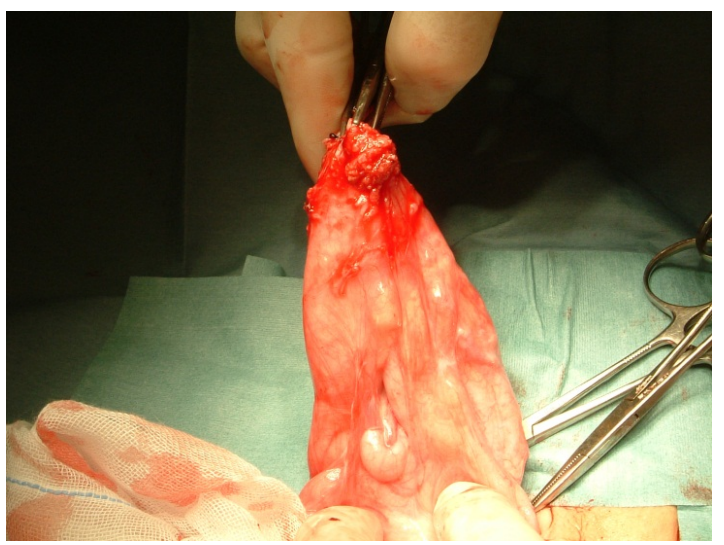

(a)

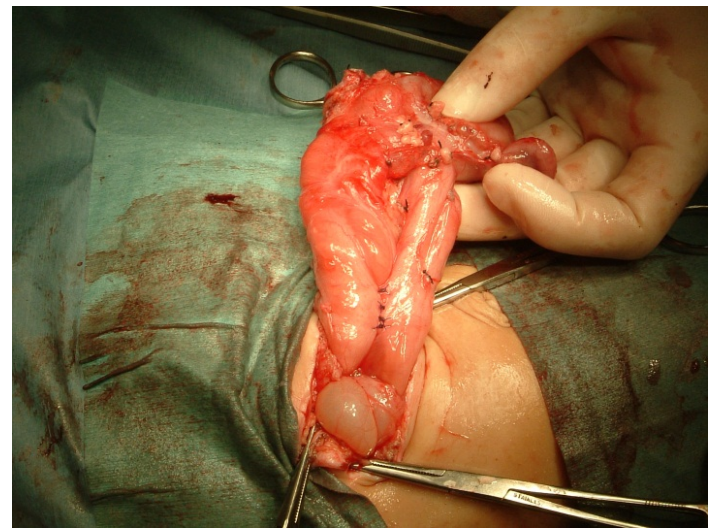

(b)

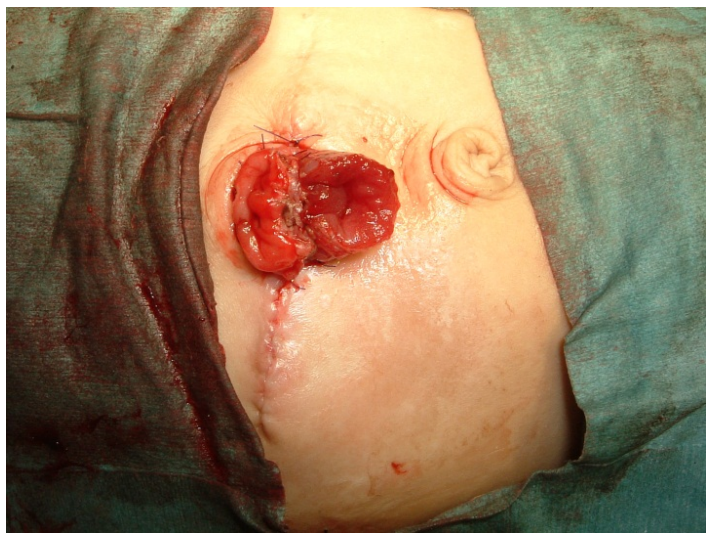

(c)

Figure 2. Operative findings at stomal reconstruction in Case 1. (a) The suture fixation to each limb of the colonic loop was loose; (b) Divided colostomy was performed with 3-cm stitching of each limb; (c) The stomal site was moved toward the middle of the body relative to the previous stomal site, with oversewing and fixation by the rectal fascia.

A radiological examination by contrast enema showed no spastic rectum and colon, as in Hirschsprung's disease. However, Hirschsprung's disease was diagnosed by an enzyme-histochemical acetylcholinesterase reaction in native mucosal biopsies of the distal rectum and the absence of a relaxation reflux by anorectal manometry. She suffered from enterocolitis 3 times since the diagnosis of Hirschsprung's disease.

Under the laparotomy of a right supra-abdominal transverse incision of $5 \mathrm{~cm}$ in diameter, loop ileostomy was performed at $30 \mathrm{~cm}$ on the proximal side of the cecum because of the presence of bowel caliber change at $10 \mathrm{~cm}$ on the proximal side of the cecum. Two centimeters of the stomal loop was approximated with sutures to prevent evisceration of the small intestine between the 2 limbs of the loop. Triangular stitches were made to the rectal fascia and peritoneum, and to each limb of the ileum loop at the point where the 2 limbs of the loop were in contact with one another. Interrupted sutures of 5-0 absorbable monofilament secured the seromuscle of the colon to the peritoneum and fascia, and also to the skin.

Subsequently, the proximal limb of the ileostomy prolapsed 2 days after operation. The physical findings revealed that $10 \mathrm{~cm}$ of the proximal limb was intussuscepted, and the intussuscepted ileum was not discolored. The stomal opening was two-fingers wide. Manual, gentle reduction of the intussuscepted ileum was successful; however, the stoma prolapsed from one to two times per day and gradually discolored (Figure 3). We performed the reconstruction of the stoma at 14 days after the operation.

For reconstruction of the stoma, a transverse abdominal incision near the medial angle of the stoma was placed in the previous wound. There was little abdominal ascites, and the suture fixation to each limb of the ileum loop was loose. Ileal resection of $40 \mathrm{~cm}$ was required for stoma-revision of the divided ileostomy because the prolapsed proximal ileum was edematous and erythematous. Divided ileostomy was performed with $3-\mathrm{cm}$ stitching of each limb (Figure 2). The stomal site was moved to the inside from the previous stomal site to oversew and fix by the rectal fascia (Figure 2). Fluids were administered

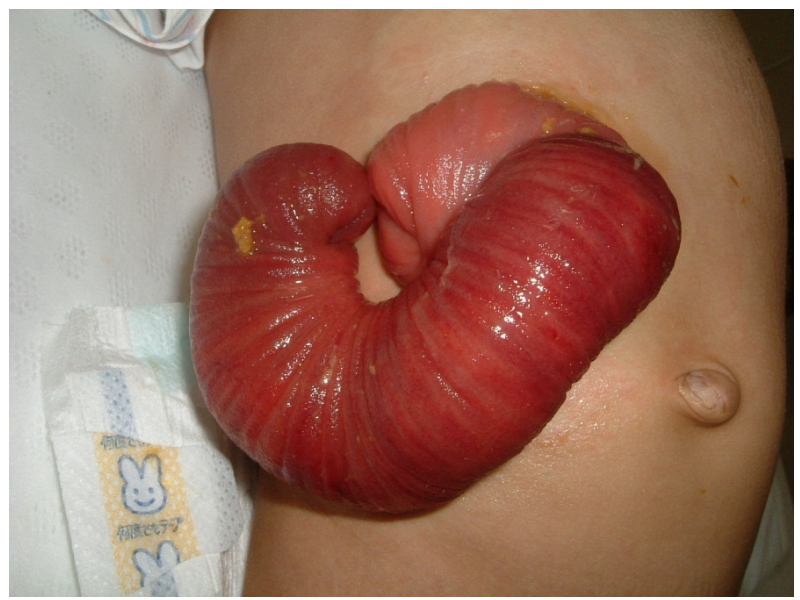

Figure 3. Appearance of incarcerated stomal prolapse in Case 2. 
by mouth for $48 \mathrm{hr}$, and thereafter, milk feedings were resumed. The child has been well without trouble since undergoing the modified Duhamel pull-through technique (Martin) at 2 years old.

\section{Discussion}

Loop colostomy is constructed mainly as a temporary diversion for stools in patients with high or intermediate anorectal malformation or Hirschsprung's disease $[7,8]$. Sigmoid loop colostomy has been shown to be associated with fewer complications compared to transverse loop colostomy [7]. It is therefore advisable to construct a sigmoid loop colostomy whenever possible. However, transverse colostomy is constructed in patients with cloacal anomalies, such as our Case 1, based on the consideration that there is insufficient distal colon for a pullthrough operation. Ileostomy is performed in patients with total colonic or extensive Hirschsprung's disease in order to facilitate bodyweight gain and prevent enterocolitis.

Stomal prolapse is defined as protrusion of at least one limb of the enterostomy for more than $6 \mathrm{~cm}$ beyond the skin surface [3]. Prolapse has more in common with intestinal intussusception than it does with hernia, and, as a corollary, is more related to point fixation and intestinal motility than to increased intra-abdominal pressure and abdominal wall laxity [3]. It was suspected that distal bowel obstruction at the time of enterostomy and loose fixation of each limb of the enteric loop were reasons for stomal prolapse through our case experience.

Stomal prolapse is the most frequently noted complication of colostomy, occurring in 3 to $8 \%$ of all colostomies, and stomas in children are more likely to prolapse than those in adults [3]. Obstruction at the time of colostomy, such as in our case of cloacal anomaly, is the all-encompassing predisposing factor of stomal prolapse. Chandler, et al. [3] reported that all but one of 11 prolapsing colostomies in children had been done to relieve distal obstruction. The mechanism appeared to be a disproportion between the size of the fascia defect and the smaller diameter of the bowel after decompression. Others have put similar stress on the size of the fascial aperture, and there is a continuing interest in techniques to make the fascia fit the bowel as snugly as possible [3]. The distal limb of any colostomy or ileostomy is destined to shrink from disuse, and therefore, is always more likely to be a loose fit in the fascia which is, why the distal unused stoma prolapses. Many authors have stressed suture fixation of the bowel at the fascial level to prevent prolapse. There is a suggestion that taking the time to fix the bowel at the fascia level may be of benefit. The loops prolapsed 10 times more frequently, $26 \%$ vs. $2.4 \%$, indi- cating a very significant difference in vulnerability to prolapse for the loop and divided types of colostomies in adults [3]. Chandler, et al. [3] reported that 6 additional operative procedures were performed in 4 of 69 patients $(6.0 \%)$ with stomal prolapse, and hastened colostomy closure occurred in 14 of 69 patients $(20.3 \%)$.

Patients are also sometimes very frustrated and depressed because of the redundant bowel and may need frequent hospital admission. Stomas in children beyond 12 months of age were particularly likely to prolapse [3]. Chandler, et al. [3] reported that the loops demonstrated a greater propensity to prolapse the more proximally they were located. However, another report found no significant difference regarding the location of the loops, whereas the prolapse rate was greater for loop than for divided colostomies [8]. According to the stomal site, the tendency for transverse colostomy to prolapse was reported previously to be 3 in 7 patients with transverse colostomy, and prolapse may have been caused by the fact that the transverse colon is relatively mobile [8]. It was more frequent in emergency stomas created for obstruction or stoma brought through the main operative incision [6].

Several special operative techniques have been described to decrease the risk of prolapse, such as skin bridge, subcutaneous tunnel for bowel exit, purse-string sutures at the fascial level, and an anchoring U stitch [6]. Kransa [4] described the simple purse-string technique for treating prolapse and intussusception of colostomy, and the results have been excellent.

Chandramouli, et al. [1] reported that revision was needed in 2 of 30 children $(6.7 \%)$ with external intestinal prolapse for significant obstructive stomal prolapse [3]. Four patients ( $6 \%$ of the prolapse group) were subjected to an extra operative procedure because of prolapse. Twice in one child and once in an adult, an attempt was made to fix the prolapse in a reduced position by button colopexy. This "spot welding" technique was unsuccessful both times in the child but held the adult's colon in place until an appropriate time for colostomy closure [3]. One attempt was made to fix the prolapsing bowel to the parietal peritoneum, using open laparotomy and multiple suture points of fixation, but this prolapse recurred. Chandramouli, et al. [1] reported that 2 prolapses were treated by advancement and amputation of the prolapsing segments, with reconstruction of the colostomy in the same location but separating the 2 limbs by a fascial bridge [3]. Al-Salem, et al. [7] reported that stomal revision was required because of frequent prolapse in 2 of 14 children who suffered prolapse. The revision rate for colostomy prolapse is lower, but when required, usually in a loop enterostomy such as in our cases, the loop is cut and converted to a divided type [1]. The stoma should 
preferably be directed through the strong muscle layer of the rectus muscle and provided with sufficient stomal limbs [6].

\section{Conclusions}

In conclusion, the creation of an abdominal stoma should not be regarded as a minor surgical procedure. Operation to repair the prolapse of a stoma is advised if it causes problems. We found that simple mobilization of the bowel and excision of the redundant bowel provided a satisfactory result in the present cases.

\section{References}

[1] B Chandramouli, K Srinivasan and N Ananthakrishnan, "Morbidity and Mortality of Colostomy and Its Closure in Children," Journal of Pediatric Surgery, Vol. 39, No. 4, 2004, pp. 596-599. doi:10.1016/j.jpedsurg.2003.12.016

[2] E. S. Golladay, F. Bernay and C. W. Wagner, "Prevention of Prolapse in Pediatric Enterostomas with Purse String Technique," Journal of Pediatric Surgery, Vol. 25,
No. 9, 1990, pp. 990-991. doi:10.1016/0022-3468(90)90244-4

[3] J. G. Chandler and B. P. Evans, "Colostomy Prolapse," Surgery, Vol. 84, No. 5, 1978, pp. 577-582.

[4] I. H. Krasna, "A Simple Purse String Suture Technique for Treatment of Colostomy Prolapse and Intussusception," Journal of Pediatric Surgery, Vol. 14, No. 6, 1979 pp. 801-802. doi:10.1016/S0022-3468(79)80269-9

[5] M. Gauderer and R. Izant, "A Technique for Temporary Control of Colostomy Prolapse in Children," Journal of Pediatric Surgery, Vol. 20, No. 6, 1985, pp. 653-655. doi:10.1016/S0022-3468(85)80017-8

[6] M. T. Cheung, "Complication of an Abdominal Stoma: An Analysis of 322 Stomas," Australian and New Zealand Journal of Surgery, Vol. 65, No. 11, 1995, pp. 808811. doi:10.1111/j.1445-2197.1995.tb00566.x

[7] A. H. Al-Salem, C. Grant and S. Khawaja, "Colostomy Complications in Infants and Children," International Surgery, Vol. 77, No. 3, 1992, pp. 164-166.

[8] N. Patwardhan, E. M. Kiely, D. P. Drake, et al., "Colostomy for Anorectal Anomalies: High Incidence of Complications," Journal of Pediatric Surgery, Vol. 36, No. 5, 2001, pp. 795-798. doi:10.1053/jpsu.2001.22963 